\title{
A Method to Take Account of Inhomogeneity in Mechanical Component Reliability Calculations
}

\author{
Jian-Ping Li and G. Thompson
}

\begin{abstract}
This paper proposes a method by which material inhomogeneity may be taken into account in a reliability calculation. The method employs Monte-Carlo simulation; and introduces a material strength index, and a standard deviation of material strength to model the variation in the strength of a component throughout its volume. The method is compared to conventional load-strength interference theory. The results are identical for the case of homogeneous material, but reliability is shown to reduce for the same load as the component volume increases. The case of a tensile bar is used to explore the variation of reliability with component volume.
\end{abstract}

Index Terms-Material inhomogeneity, mechanical reliability, Monte Carlo.

\section{ACRONYMS $^{1}$}

FMECA Failure Mode, Effect, and Criticality Analysis

FTA Fault Tree Analysis

SDH Standard Deviation of material strength related to material inHomogeneity

SDQ Standard Deviation of material strength related to material Quality stability

MSI Material Strength Index

\section{NOTATION}

$s \quad$ Stress of a component

$\delta \quad$ Material strength of a component

$s_{1}, \quad$ Principal normal stresses

$s_{2}, s_{3}$

$s_{t}, s_{c}$

Uniaxial failure strength in tension, and in compression

$\mu_{s}, \quad$ Mean value, and standard deviation of the material

$\sigma_{s} \quad$ stress $(s)$ of a component

$\mu_{\delta}$, Mean value, and standard deviation of the material

$\sigma_{\delta} \quad$ strength $(\delta)$ of a component

$N\left(\mu, \sigma^{2}\right) \quad S$-normal distribution with mean value $\mu$, and standard deviation $\sigma$

Manuscript received August 4, 2003; revised October 1, 2004. This work was supported by the U.K. Engineering \& Physical Science Research Council. Associate Editor: C. Smith.

J.-P. Li is also with the Applied Mechanics Division, Department of Mechanical, Aerospace and Manufacturing Engineering, UMIST, Manchester, M60 1QD, U.K. and the Department of Management Engineering, Shijiazhuang Institute of Science \& Technology, Shijiazhuang, 050003, P.R. China (e-mail: Jian-Ping.Li@umist.ac.uk; ljp218@ @otmail.com).

G. Thompson is with the Applied Mechanics Division, Department of Mechanical, Aerospace and Manufacturing Engineering, UMIST, Manchester, M60 1QD, U.K. (e-mail: graham.thompson@umist.ac.uk).

Digital Object Identifier 10.1109/TR.2004.837702

${ }^{1}$ The singular and plural of an acronym are always spelled the same. $\mu_{h}, \quad$ Mean value, and standard deviation of material $\sigma_{h} \quad$ strength related to material inhomogeneity $\mu_{q}, \quad$ Mean value, and standard deviation of material $\sigma_{q} \quad$ strength related to material quality stability $\alpha \quad$ Material strength index (MSI)

$\mu_{l}, \sigma_{l} \quad$ Mean value, and standard deviation of bar' load or force

$l \quad$ Length of the bar

A Area

$r \quad$ Radius

$\mu_{r}, \quad$ Mean value, and standard deviation of bar's radius $r$

$\sigma_{r}$

$\mu_{A}, \quad$ Mean value, and standard deviation of bar's cross

$\sigma_{A} \quad$ area $A$

$V \quad$ Volume of a component

$V_{s} \quad$ Volume of the test specimen, which is used to test material strength

$R \quad$ Reliability of a mechanical component

$F \quad$ Failure probability or cumulative function of a failure

$\underline{N} \quad$ A set of integer number from 1 to $N$

$N_{s} \quad$ Total number of simulations

$N_{F} \quad$ Number of the component failures or of successful simulations

$N_{v} \quad$ Number of the selected points related on the component's volume

E A random event

$I(s, \delta) \quad$ A failure indicator function, $I(s, \delta)=1$ if there is a failure $(s>\delta)$, or $I(s, \delta)=0$

\section{INTRODUCTION}

$\mathbf{R}$ ECENTLY, much progress has been made in reliability engineering research, and applications, since Bazovski [1] identified the reliability approach based on risk assessment. For electronic components and products, there are mature reliability theories and techniques. The history of risk or probability assessment has been considered in some detail by Moss [2], who has described the background and relevance of now well-established methods, such as Failure Mode Effect and Criticality Analysis (FMECA), and Fault Tree Analysis (FTA). Moss [2] also pointed out that there is some difficulty with mechanical reliability prediction, and that the techniques developed many years ago for evaluation of electronic systems and components must be used with some caution when dealing with mechanical systems.

Failure is a fundamental concept of any reliability analysis [3]. Mechanical failure may be defined as any change in size, shape, or material properties of a structure, machine, or machine 


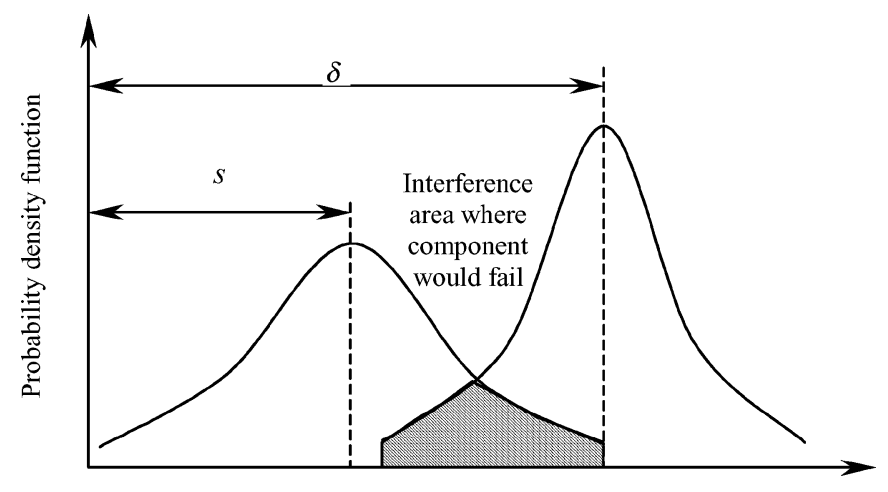

Strength and stress measured in identical units

Fig. 1. Load and strength interference diagram with interference.

part which renders it incapable of satisfactorily performing its intended function [4].

In the literature, there are two main approaches used in engineering design to analyze and predict mechanical failures: stress failure theory, and reliability theory.

Collins [4] described six stress failure theories:

1) Maximum normal stress theory,

2) Maximum shearing stress theory,

3) Maximum normal strain theory,

4) Total strain energy theory,

5) Distortion energy theory, and

6) Mohr's failure theory.

With the exception of composite materials, the basic assumptions of all these failure theories are that the material is homogenous, and that a failure is predicted to occur when the failure criterion is satisfied. A safety factor is then applied based on experience, design code, or an analysis of the worst loading conditions [5]. These methods do not explicitly take into account variations in component material strength due to variations from construction and manufacture. The application of a safety factor takes into account a wide range of uncertainties including the applied loads and variations in material strength.

An alternative approach is reliability theory [6], [7]. The loadstrength interference theory [8]-[13] is a well-known method to calculate the reliability of mechanical components. This early work was carried out by Freudenthal et al. [8], with respect to the safety of structures. The method was also applied to design of critical mechanical component by Kececioglu and Cormier [14].

The probability density functions for both strength and load are shown in Fig. 1, and the shaded area where the curves interfere is an indication that the component will fail. Load-strength interference theory has been used to take into account variations in the operating load and strength of mechanical elements [8], [12] which are distributed about a mean value, and which may change with time in service operation owing to degradation. Variation in the strength of the component may result from a combination of variations in the metallurgical and fabrication processes involved with its manufacture, dimensional inaccuracy, surface damage, etc.; whereas variation in the load imposed upon it may result from variations in its duty, and environment. The bigger the difference $(|\delta-s|)$ between strength, and stress, as shown in Fig. 1, the higher the reliability of the compo-

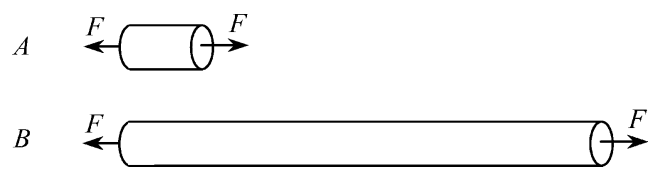

Fig. 2. Bars with different lengths.

nent becomes. The use of a safety margin in design calculations is attractive, but it depends upon there being sufficient information available about strength, and load distributions [15]. The safety factor design approach introduces the safety factor to enlarge the difference between strength, and load.

The strength of a component at different points will not remain constant because defects are introduced during material processing, and component manufacture. It is widely recognized by engineers that failure occurs in local 'weak spots'.

Consider the case of two bars, A and B, which have the same cross section, and the same applied load, but they have different lengths, as shown in Fig. 2. Conventionally, both bars would have the same stress, the same material strength, and the same probability of failure, i.e., they have the same reliability. However, in practice, a long bar is often found to be less reliable than a shorter one [16].

The aim of this paper is to explore the relationship between the physical size of a mechanical component, and its reliability.

A new method is proposed below by which material inhomogeneity may be modeled in a component reliability calculation. The strength of a component depends upon the quality of the materials used, and the quality of construction and manufacturing processes employed.

The new method employs the Monte-Carlo simulation method, which uses system probability models, and simulation of random variables [17], to analyze the relationship between the reliability, and volume, of a mechanical component.

A comparison is made with two existing calculation methods, the load-strength interference method, and the use of a stress based failure criterion plus safety factor, with the new method presented in this paper.

\section{MechaniCAL ReLiabiLITy}

\section{A. Mechanical Failure}

The maximum normal stress theory [4] is used to define mechanical failures in this paper:

Failure is predicted to occur in the multiaxial state of stress when the maximum principal normal stress becomes equal to or exceeds the maximum normal stress at the time of failure in a simple uniaxial stress test using a specimen of the same material.

A failure is predicted by this theory to occur if any one expression is satisfied

$$
\left|s_{i}\right| \geq|\delta| \quad\left(\mathrm{i}=1,2,3 \quad \delta=\delta_{t} \text { or } \delta_{c}\right)
$$

where $s_{1}, s_{2}$, and $s_{3}$ are the principal normal stresses; $\delta$ could be the uniaxial failure strength in tension $\left(\delta_{t}\right)$ or compression $\left(\delta_{c}\right)$.

Of course, this is not the only failure theory which could be used in the new method, and other failure theories [4] could be adopted too. 

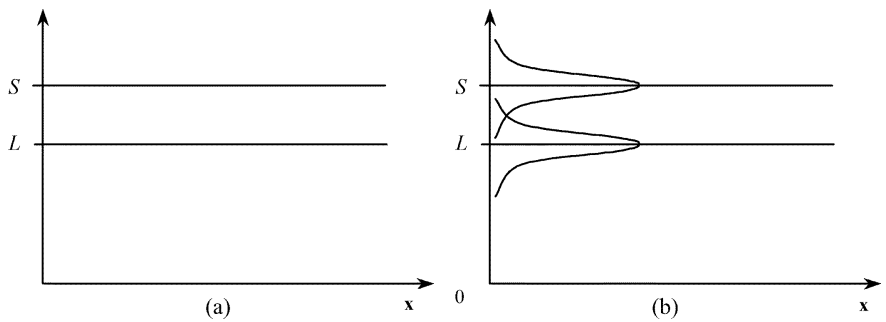

Fig. 3. Strength variation in different failure theories.

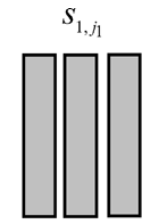

Batch 1

$s_{1, j,}, j_{1} \in N_{1}$

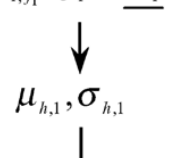

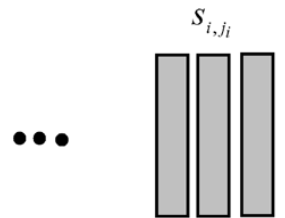

Batch $i$

$s_{i, j i}, j_{i} \in N_{i}$

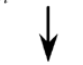

$\mu_{h, i}, \sigma_{h, i}$

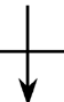

$\mu_{q}, \sigma$
Fig. 4. Samples from different batches in a strength test.

\section{B. Variation of Material Strength}

From (1), there are two factors: principal normal stresses $\left(s_{i}\right)$, and the material strength $(\delta)$, which determine if a mechanical failure occurs.

The stress failure theory plus safety factor method assumes that the stresses and strengths remain constant in any environment with repeated load, as shown in Fig. 3(a). For example, in the case of a bar subject to an axial load, the load induced stresses and material strength at any point in the bar are thought to be the same. In the load-strength interference theory [8], there are variations in both load and strength, as shown in Fig. 3(b). Variation in the strength of the component may result from a combination of variations in the metallurgical and fabrication processes involved with its manufacture, whereas variations in the load result from variations in its duty and environment.

To analyze the variation in the material strength, a method to calculate the mean value, and standard deviation, of material strength from test data is first investigated. It is assumed that test samples come from different batches, and $s_{i, j_{i}}$ denotes the strength of Sample $j_{i}$ in Batch $i$. The number of the samples in Batch $i$ is $N_{i}$. There are $n$ batches in the strength test shown in Fig. 4.

The total number of the samples in the test, $N$, is

$$
N=\sum_{i=1}^{n} N_{i}
$$

Then the mean value and standard deviation of the material strength could be calculated as

$$
\begin{aligned}
& \mu_{\delta}=\frac{1}{N} \sum_{i=1}^{n} \sum_{j_{i}=1}^{N_{i}} s_{i, j_{i}} \\
& \sigma_{\delta}^{2}=\frac{1}{N-1} \sum_{i=1}^{n} \sum_{j_{i}=1}^{N_{i}}\left(s_{i, j_{i}}-\mu_{\delta}\right)^{2} .
\end{aligned}
$$

Here, another method is introduced to compute them. First, the mean value, and standard deviation, of the material strength in Batch $i$ are calculated respectively as

$$
\begin{aligned}
& \mu_{h, i}=\frac{1}{N_{i}} \sum_{j_{i}=1}^{N_{i}} s_{i, j_{i}} \\
& \sigma_{h, i}^{2}=\frac{1}{N_{i}-1} \sum_{j_{i}=1}^{N_{i}}\left(s_{i, j_{i}}-\mu_{h, i}\right)^{2} .
\end{aligned}
$$

Then the mean value, and standard deviation, of the material can be calculated as:

$$
\begin{aligned}
& \mu_{q}=\frac{1}{n} \sum_{i=1}^{n} \mu_{h, i} \\
& \sigma_{q}^{2}=\frac{1}{n-1} \sum_{i=1}^{n}\left(\mu_{h, i}-\mu_{q}\right)^{2} .
\end{aligned}
$$

The results obtained from the above two methods should be different. The mean of the material strength in (3) will be the same with the one in (7) only when there are the same number of samples in each batch.

For given batch samples, the manufacturing condition, and the material ingredient, could be thought to be the same. The material is thought to be homogeneous if each sample has the same strength, that is

$$
\sigma_{h, i}=0 .
$$

Actually, it is impossible that the strength of each sample is kept the same. This means that the material is inhomogeneous.

Next, consider another situation. It is assumed that the material is homogeneous in each batch. Other factors will affect the material strength, such as the variation of material ingredients, and the manufacturing control parameters. There are different strengths in different batches. Then the deviation of material strengths also exists.

So two known methods to reduce the deviation of material strength are:

1) raising the homogeneity of the material (to reduce $\sigma_{h, i}$ ), and

2) controlling the stability of material quality in different batches (to reduce $\sigma_{q}$ ).

Based on the above brief discussion, there are two factors which affect the variation of the strength of a material:

1) the material inhomogeneity (or imperfects), and

2) the material quality stability.

Only the more homogenous and more stable the material quality becomes, then the less the variation there is in material strength.

Two deviations of material strength are introduced:

1) the standard deviation of material strength related to material inhomogeneity $(S D H)$, and

2) the standard deviation of material strength related to material quality stability $(S D Q)$.

Respectively, they are denoted as $\sigma_{h}$ and $\sigma_{q}$, and then we have the following two distributions:

1) a distribution of material strength related to material inhomogeneity $N\left(\mu_{h}, \sigma_{h}^{2}\right)$, and

2) a distribution of material strength related to material quality stability $N\left(\mu_{q}, \sigma_{q}^{2}\right)$. 


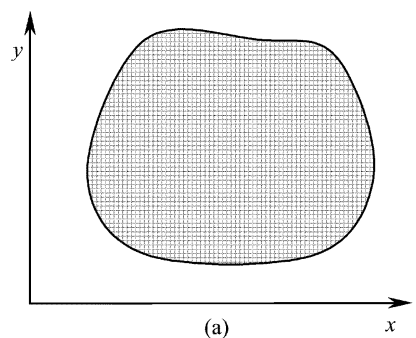

(a)

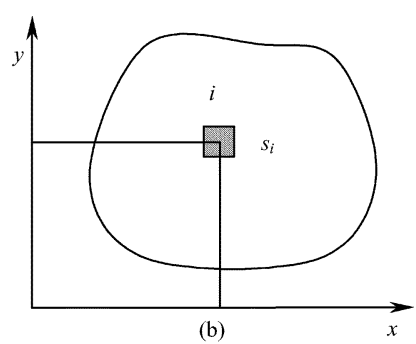

(b)

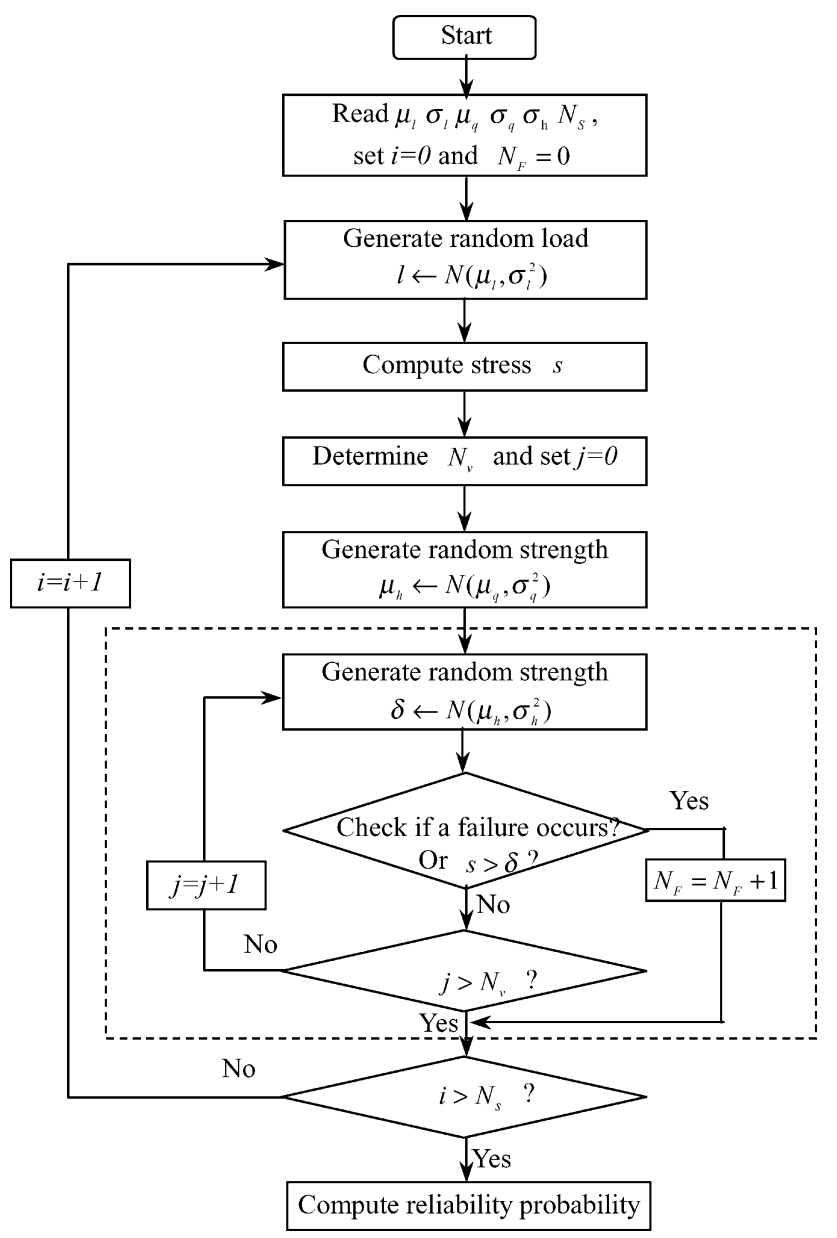

Fig. 6. Flowchart of the Monte-Carlo simulation for mechanical reliability.

\section{Monte-CARlo Simulation}

\section{A. Monte-Carlo Simulation Flow}

Monte Carlo simulation is often employed in reliability analysis when the analytical solution is not attainable, and the failure domain cannot be expressed or approximated by an analytical form. The flow of the Monte Carlo simulation for evaluating the effect of material inhomogeneity and material quality stability on the reliability of mechanical components is shown in Fig. 6.

If the proportion in the dashed area in Fig. 6 is removed, it is just a general Monte-Carlo simulation for determining the reliability of mechanical components. The task of the enclosed dashed area is to simulate the effect of the material inhomogeneity on mechanical reliability.

The randomness of random variables can be described by the known probability density function of its distribution. For example, the random load $l$ will be calculated by using its probability density function of the s-normal distribution $N\left(\mu_{l}, \sigma_{l}\right)$.

The main steps of the Monte-Carlo simulation technique as shown in Fig. 6 are:

Step 0: Read input data $\mu_{l}, \sigma_{l}, \mu_{q}, \sigma_{q}, \sigma_{h}, N_{s}$; Let $N_{F}=0$, $i=0$;

Step 1: Generate a random load, $l$, from the distribution $N\left(\mu_{l}, \sigma_{l}^{2}\right)$;

Step 2: Calculate the stress $s$ by using Finite-Element tools, or mechanics methods. 
Step 3: Generate a random strength.

3.1: Compute the selected number, $N_{v}$, of the points which are needed to generate random strength. Let $j=0$;

3.2: Determine the mean value of material strength related to material inhomogeneity, $\mu_{h}$, from the distribution $N\left(\mu_{q}, \sigma_{q}^{2}\right)$

3.3: Generate the random strength, $\delta$, from the distribution $N\left(\mu_{h}, \sigma_{h}^{2}\right)$;

3.4: If $s>\delta$, a failure happens, $N_{F}=N_{F}+1$, and then go to step 4 .

3.5: If $j<N_{v}$, set $j=j+1$, and return to step 3.2.

Step 4: $i=i+1$; if $i<N_{s}$, return to Step 1 .

Step 5: Calculate the reliability of the component, and stop.

Following the law of large numbers, an unbiased estimator of the failure probability of the component is given by

$$
F=\frac{1}{N_{s}} \sum_{i=1}^{N_{s}} I\left(l_{i}, s_{i}\right)=\frac{N_{F}}{N_{s}}
$$

where $I\left(s_{i}, \delta_{i}\right)$ is an indicator defined as:

$$
I\left(s_{i}, \delta_{i}\right)=\left\{\begin{array}{ll}
1 & \text { if } \mathrm{s}_{i}>\delta_{i} \\
0 & \text { if } \mathrm{s}_{i} \leq \delta_{i}
\end{array} .\right.
$$

The reliability of the component is given by

$$
\begin{aligned}
R & =1-F \\
& =\frac{1}{N_{s}} \sum_{i=1}^{N_{s}} I\left(s_{i}, \delta_{i}\right) .
\end{aligned}
$$

\section{B. Number of Selected Points, $N_{v}$}

Section II-B shows that the inhomogeneity will affect the strength in the material. The bigger the volume of a component is, then the greater the effect will be.

To investigate the effect of the inhomogeneity on reliability, a new number, $N_{v}$, is introduced to describe the relationship between the volume of the component, and its reliability. In Fig. 6, the load, and $\mu_{h}$, are randomly generated according to their respective probability density functions. Then the program generates a range of component strengths according to the material strength related to the distributions $N\left(\mu_{q}, \sigma_{q}^{2}\right)$, and $N\left(\mu_{h}, \sigma_{h}^{2}\right)$. This means that there are several points, $N_{v}$, which are selected, to check if a failure occurs.

The number of selected points, $N_{v}$, is a function of the volume of a component. Generally, $N_{v}$ increases with the volume of the component. Here the linear relationship is supposed. This is given by

$$
N_{v}=\alpha \cdot V_{c}
$$

where $V_{c}$ is the volume of the component, and $\alpha$ is defined as Material Strength Index(MSI).

In this paper, Material Strength Index $\alpha$ is set as a constant. If there is only one point selected

$$
N_{v}=1
$$

then the result should be equal to the result obtained from the load-strength interference theory.

The number of selected point, $N_{v}$, will be an integer. But $N_{v}$ from (19) is a real number. For example, if $N_{v}=14.55$, then there are two methods to set $N_{v}$ : one is that $N_{v}=14$, and another is that $N_{v}=15$. Here a random method is introduced. Function $\operatorname{int}\left(N_{v}\right)$ returns the integer part of $N_{v}$, thus returning 14. First, a random real number $r$ is generated, which is in $[0$, 1]. If $r$ is less than or equal to $N_{v}-\operatorname{int}\left(N_{v}\right)$, then $N_{v}$ is set to $\operatorname{int}\left(N_{v}\right)$, or $N_{v}$ is set to $\operatorname{int}\left(N_{v}\right)+1$. This is

$$
N_{v}=\left\{\begin{array}{ll}
\operatorname{int}\left(N_{v}\right) & \text { if } r \leq N_{v}-\operatorname{int}\left(N_{v}\right) \\
\operatorname{int}\left(N_{v}\right)+1 & \text { if } r>N_{v}-\operatorname{int}\left(N_{v}\right)
\end{array} .\right.
$$

\section{Selection of Material Strength Index}

The MSI, $\alpha$, has a strong effect on the results obtained by using the method shown in Fig. 6. The material strength index should be calculated carefully as follows.

Ordinarily, mechanical properties are obtained from experimental results. So that test results will be comparable, the dimensions of test specimens, and the methods of applying loads, have been standardised. One of the major standards organizations is the American Society for Testing and Material (ASTM). The ASTM standard tension specimen has a diameter of 0.505 inches, and a gauge length of 2.0 inches [18]. This means that the volume of the specimen is

$$
\begin{aligned}
V_{c} & =\pi \times\left(\frac{0.505}{2}\right)^{2} \times 2.0=0.40059225\left(\mathrm{in}^{3}\right) \\
& =6564.53083701(\mathrm{~mm})^{3} .
\end{aligned}
$$

It is supposed the reliability of the standard specimen obtained from the above Monte-Carlo simulation method is equal to the one calculated by using the load-strength interference theory. This implies that the number of selected point $N_{v}$ in Fig. 6 should be equal to 1. From (19), we have

$$
N_{v}=\alpha \cdot V_{c}=1 \text {. }
$$

Then

$$
\begin{aligned}
\alpha & =\frac{1}{V_{c}}=\frac{1}{6564.53083701} \\
& =1.523338110 \times 10^{-4}\left(\frac{1}{\mathrm{~mm}^{3}}\right) .
\end{aligned}
$$

If the volume of a component is less than the one of the standard specimen, the number of selected points $N_{v}$ that are obtained from (21) could be zero or one. At that time, the component reliability will be greater than the standard specimen. When the volume is very small, and approaches zero, the reliability approaches one because there is no volume in which material defects can occur. This is

$$
R \approx 1, \quad \forall V_{c} \approx 0 .
$$

This may be true, but it should be proved by the test and practise. Here, conservatively let

$$
N_{v} \geq 1 \text {. }
$$

Equation (26) means that when the volume of a component is less than that of the standard specimen, which is used to determine the material strength, its reliability or failure probability is the same with the one of the standard specimen.

The above method to determine the Material Strength Index will not affect the Monte Carlo simulation method presented in 
this paper. If the strength comes from different standard specimens, the material strength index $\alpha$ should be modified with the size of the specimens.

\section{EXAMPLES AND ANALYSIS}

The cases of bars subject to tensile loading are used to analyze the effect of material inhomogeneity on mechanical reliability. The parameters investigated in this paper using the proposed analysis method are:

- standard deviation of material strength related to material inhomogeneity,

- applied load, and

- bar length and radius.

In this section, all the simulation results were averaged over 50 runs, and in each run the simulation number was set to 1000 .

\section{A. Theoretical Reliability}

Here the conventional load-strength interference theory is used to calculate the bar theoretical reliability. Let $r$ be the bar radius, and $l$ be the bar length. It is assumed that the distributions of all the random variables are $s$-normal distributions. The distribution of the cross area of the bar is also the $s$-normal distribution $N\left(\mu_{A}, \sigma_{A}^{2}\right)$, its mean value is

$$
\mu_{A}=\pi \cdot \mu_{r}^{2}
$$

and the cross-area standard deviation is

$$
\sigma_{A}=2 \pi \cdot \mu_{r} \cdot \sigma_{r}
$$

The stress distribution is $N\left(\mu_{s}, \sigma_{s}^{2}\right)$, and its mean value is

$$
\mu_{s}=\frac{\mu_{l}}{\mu_{A}}=\frac{\mu_{l}}{\pi \cdot \mu_{r}^{2}} .
$$

Here $l$ is the load or force, $\mu_{l}$ is its mean value, and $\sigma_{l}$ is its standard deviation. The standard deviation of the stress is written as

$$
\sigma_{s}=\frac{1}{\mu_{A}^{2}} \sqrt{\mu_{l}^{2} \cdot \sigma_{A}^{2}+\mu_{A}^{2} \cdot \sigma_{l}^{2}} .
$$

According to the load-strength interference theory, the theoretical reliability of the bar is

$$
R=G\left(\frac{\mu_{\delta}-\mu_{s}}{\sqrt{\sigma_{\delta}^{2}+\sigma_{s}^{2}}}\right)
$$

where $\mu_{\delta}$, and $\sigma_{\delta}$ are respectively the mean value, and standard deviation of material strength. Here, in the load-strength interference theory, the material is supposed to be homogeneous, so we have

$$
\mu_{\delta}=\mu_{q}
$$

and

$$
\sigma_{\delta}=\sigma_{q}
$$

TABLE I

PARAMETERS IN EXAMPLE 1

\begin{tabular}{c|c|c}
\hline & Mean value & Standard deviation \\
\hline Load (tension force) (N) & 80,000 & 3,000 \\
\hline $\begin{array}{c}\text { Material strength related to material quality } \\
\text { stability (MPa) }\end{array}$ & 660 & 25 \\
\hline $\begin{array}{c}\text { Material strength related to material } \\
\text { inhomogeneity (MPa) }\end{array}$ & ---- & 12.5 \\
\hline Bar radius (mm) & 6.4135 & 0.0320675 \\
\hline Bar length (mm) & 50.8 & ---- \\
\hline
\end{tabular}

TABLE II

COMPARISON BETWEEN THEORETICAL RELIABILITY AND THE SiMULATION RESUlT

\begin{tabular}{l|c|c}
\hline & Bar reliability & Variation \\
\hline Load-strength interference theory & 0.881000 & - \\
\hline \multicolumn{1}{c}{ Monte-Carlo simulation } & 0.880594 & 0.000406 \\
\hline
\end{tabular}

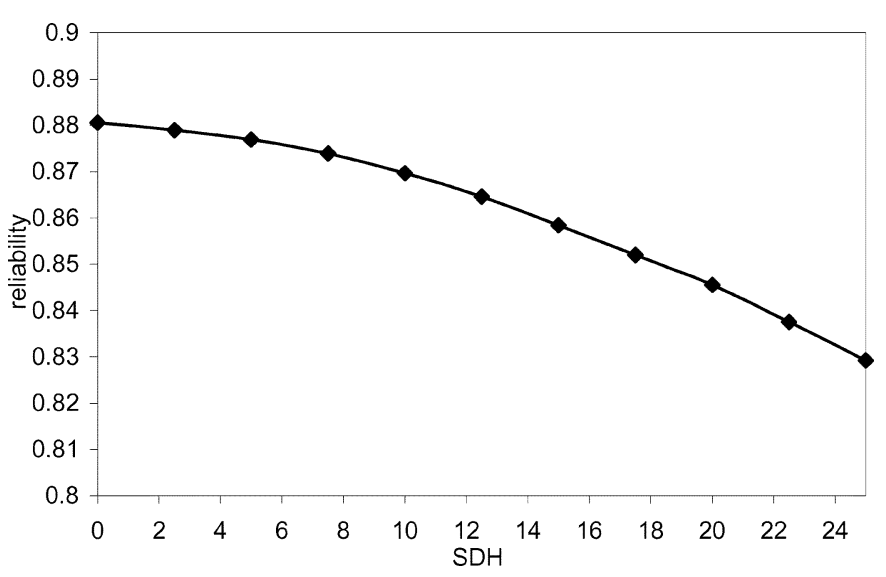

Fig. 7. Variation in reliability with the $S D H$.

The reference data used in the example calculation is given in Table I. The radius of the tensile specimen is set to be the same as the one of the standard specimen, thus its mean value $\mu_{r}$ is $6.4135(\mathrm{~mm})$, and its standard deviation $\sigma_{r}$ is set in all examples according to the following equation:

$$
\sigma_{r}=0.005 \mu_{r}
$$

From (34) the theoretical reliability of the bar is $R_{t}=$ 0.88100 . The result obtained by the Monte-Carlo simulation program, which is used in the new method, is $R_{t}=0.880594$, is shown in Table II. The variation of the bar reliability from the load-strength interference theory, and from the new method, is only $0.046 \%$. Therefore, the program used calculates the theoretical reliability of the bar with high accuracy.

\section{B. Performance of Standard Deviation of Material Strength Related to Material Inhomogeneity}

To investigate the effects of the standard deviation of material strength related to material inhomogeneity $\left(\mathrm{SDH}, \sigma_{h}\right)$ on the reliability, modeling experiments were carried out in which $\sigma_{h}$ was varied, and the results obtained are shown in Fig. 7. As expected, when $\sigma_{h}$ is increased, the bar reliability decreases. When $\sigma_{h}$ varies from 0 to 25 , which is equal to $\sigma_{q}=25$ (SDQ) in 


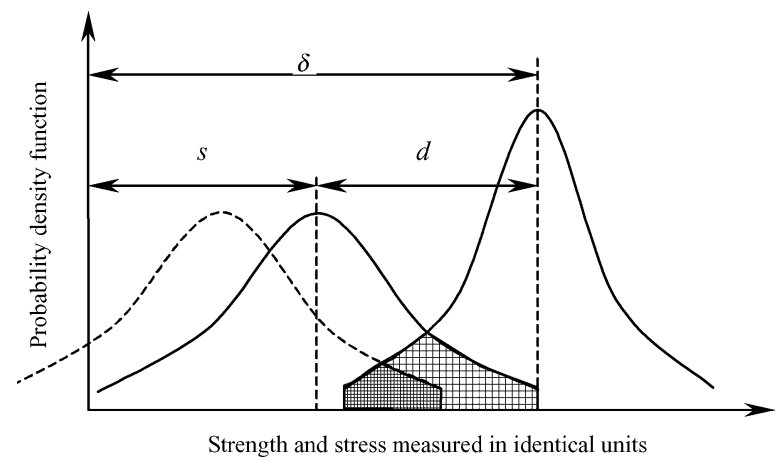

Fig. 8. Effect of stress and strength on the reliability.

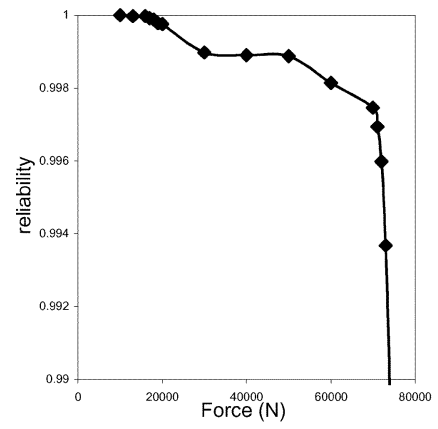

(a)

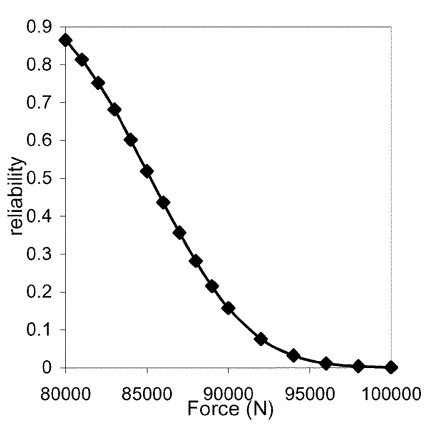

(b)
Fig. 9. Performance of load or force on reliability.

this case, the bar reliability drops from 0.880594 to 0.829198 . It is interesting that when $\sigma_{h}$ is set to zero, the reliability is equal to the theoretical reliability 0.881000 obtained by using the load-strength interference theory, which is shown in Table II. This means that, if the material is homogeneous, the result found by using the new method is the same as the one calculated with the load-strength interference theory. If material is inhomogeneous, the reliability obtained through the load-strength interference theory is higher.

\section{Effects of Applied Loads and Bar Radius on Reliability}

According to the load-strength interference theory, the stress distribution of a component, and the material strength distribution determine its reliability. The difference between the stress, and strength is written as

$$
d=\mu_{\delta}-\mu_{s} .
$$

The smaller the value of $d$, the larger the shaded area becomes, as in Fig. 8, and the higher the failure probability of the component. The reliability decreases as the load (applied stress) increases.

There are two factors which affect the stress: the load, and the radius of the bar. With the same parameter settings, which are shown in Table I (except the load, and radius variations) the results obtained by using the new Monte-Carlo simulation method in Section III are shown in Figs. 9 and 10.

In Fig. 9, the load varies from 0 to 100000 N. The load varies between 0 and $80000 \mathrm{~N}$ in Fig. 9(a), and from $80000 \mathrm{~N}$ to $100000 \mathrm{~N}$ in Fig. 9(b). Fig. 9 shows that, as the load increases, the bar reliability decreases. The reason is, of course, that the increase of the load $(l)$ will results to the increase of the stress

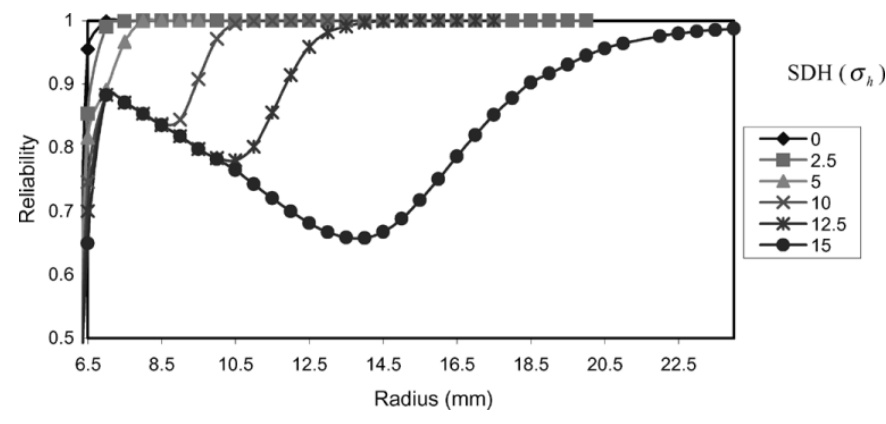

Fig. 10. Performance of radius and $\sigma_{h}$ on reliability.

and the decrease of the distance between the stress and its strength in (35), $d$ so that the shaded area increases. Therefore there are more opportunities for a failure to occur, and the reliability will decrease.

Fig. 10 illustrates the relationship between the bar reliability and the bar radius, and the standard deviation of material strength related to material inhomogeneity $\sigma_{h}$. The bar radius varies from 6.5 to $24 \mathrm{~mm}$, and $\sigma_{h}$ from 0 to $15 \mathrm{MPa}$.

The reliability rises sharply because the cross sectional area increases dramatically with the square of the radius, and the stress reduces accordingly. However, an interesting thing happens when the bar radius is larger than $7 \mathrm{~mm}$ in this case. If $\sigma_{h}$ is less than $5 \mathrm{MPa}$, the reliability increases rapidly, and remains constant at the top level when the radius is bigger than $10(\mathrm{~mm})$. However, when $\sigma_{h}$ is larger than $5(\mathrm{MPa})$, the reliability drops, and then increases until it reaches unity.

A possible explanation is that the radius has appeared to have two different effects on reliability. The first one is that when the radius increases, the stress drops sharply and so the reliability rises. However, the increase of the bar radius results in the increase of the bar volume, which leads to more opportunities for a failure to occur. The increase in bar volume reduces reliability. The decrease in reliability means that the volume has a strong effect on failures due to the higher value of $\sigma_{h}$. The increase of the bar radius results in the stress pdf to move to the left, and the shaded area in Fig. 11 will decrease, and thus increase reliability. However, the increase of $\sigma_{h}$ will let the strength pdf become wide, so that the shaded area will increase, and then the reliability decreases. The two factors lead to the behaviors shown in Fig. 10.

\section{Effects of Bar Length on Reliability}

Figs. 12 and 13 show the relationship between the reliability, and the bar length. The bar length varies between 50.8 and 110 (mm) in Fig. 12, and from 0 to 1000 times of the length of the standard specimen in Fig. 13. Other parameters remain constant as given in Table I. The specimen length used is $50.8 \mathrm{~mm}$ (2in).

For bars with a length greater than $50.8 \mathrm{~mm}$, the bar volume is bigger than the specimen volume. Therefore, due to the variation in the material strength related to material inhomogeneity, the failure probability rises, and so the reliability decreases with the increase of the bar length. Fig. 12 shows the decrease in bar reliability as the bar length (and hence volume) increases.

Fig. 13 shows how reliability varies with the bar length and $\mathrm{SDH}$. The increase of the bar length results in the increase of its 


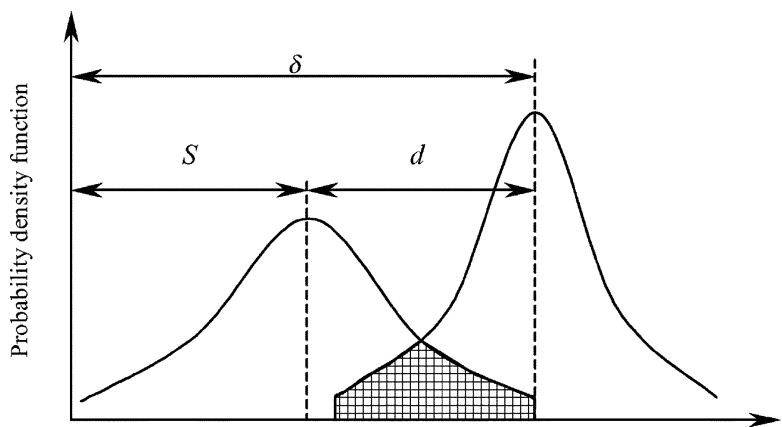

Strength and stress measured in identical units

(a) the original chart

Fig. 11. Effects of bar radius and $\sigma_{h}$ on the reliability.

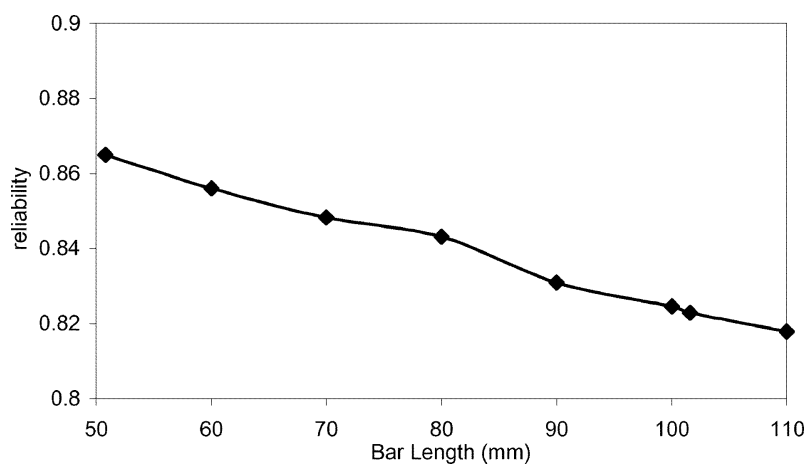

Fig. 12. Performance of the bar's length.

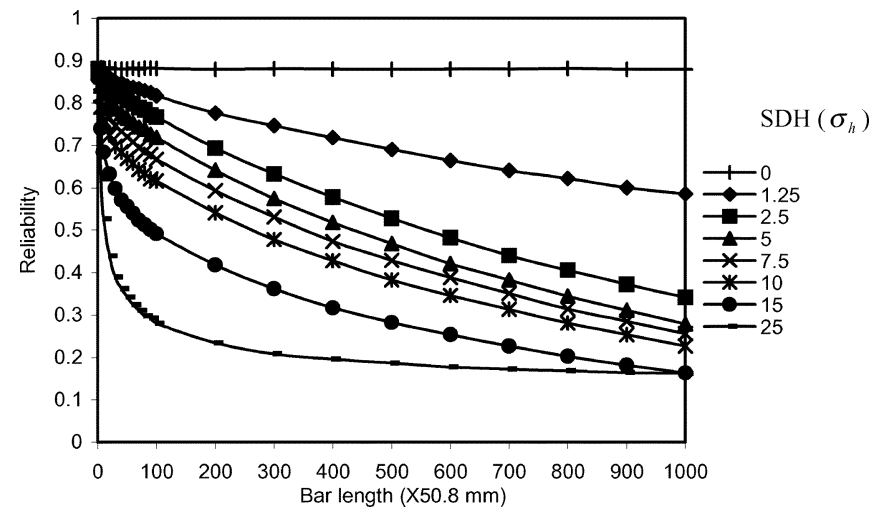

Fig. 13. Performance of the bar's length.

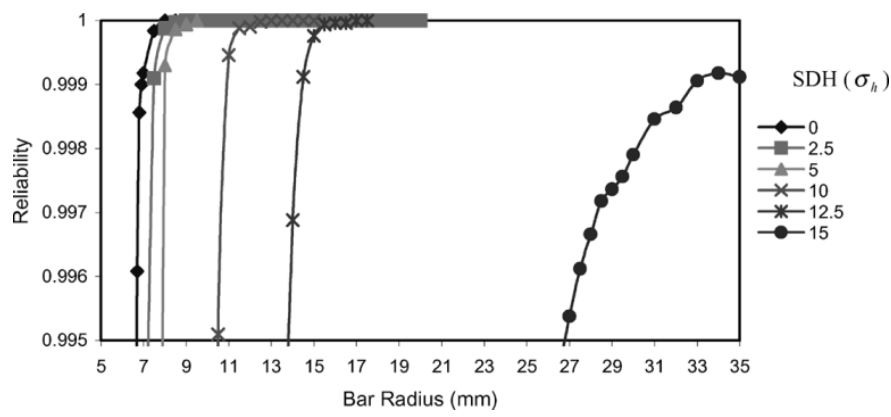

Fig. 14. Effects of the radius and $\sigma_{h}$ on reliability.

volume, and then its reliability decreases. The SDH also results in the decrease of the reliability.

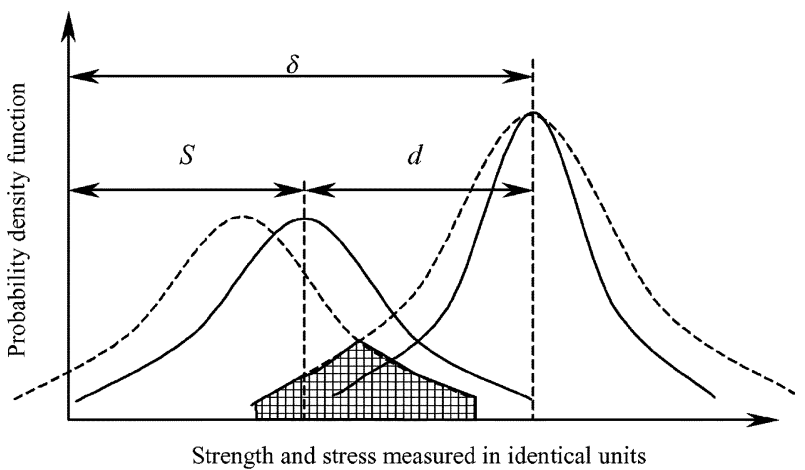

(b) the new chart

When the SDH is equal to zero, $\sigma_{h}=0$, the reliability remains constant at 0.88 . This means that, if the material is homogeneous, the reliability does not vary with the increase of the volume of a component, and its reliability is equal to the one calculated by using the conventional Load-Strength interference theory.

\section{E. Bar Structure Design}

It is assumed that a bar of length is $5000 \mathrm{~mm}$, and that the reliability of the bar must be greater than or equal to 0.999

$$
R \geq 0.999 .
$$

If the applied loads are as shown in Table I, what is the radius of the bar?

1) Design Using the Load-Strength Interference Theory: Using the load-strength interference theory, the mean value, and standard deviation, of the stress are written separately as

$$
\begin{aligned}
\mu_{s}= & \frac{\mu_{l}}{\mu_{A}}=\frac{80000}{\pi \cdot \mu_{r}^{2}}=\frac{25464.79619}{\mu_{r}^{2}} \\
\sigma_{s}= & \frac{1}{\mu_{A}^{2}} \sqrt{\mu_{l}^{2} \cdot \sigma_{A}^{2}+\mu_{A}^{2} \cdot \sigma_{l}^{2}} \\
= & \frac{1}{\left[\pi \cdot \mu_{r}^{2}\right]^{2}} \\
& \bullet \sqrt{(80000)^{2}\left[2 \pi\left(0.005 \mu_{r}\right) \mu_{r}\right]^{2}+\left[\pi \mu_{r}^{2}\right]^{2}(3000)^{2}} \\
= & \frac{988.29986}{\mu_{r}^{2}} .
\end{aligned}
$$

The bar reliability is calculated by using the following equation:

$$
R=G\left(\frac{\mu_{\delta}-\mu_{s}}{\sqrt{\sigma_{\delta}^{2}+\sigma_{s}^{2}}}\right)=0.999
$$

Thus,

$$
\begin{aligned}
z_{R} & =\frac{\mu_{\delta}-\mu_{s}}{\sqrt{\sigma_{\delta}^{2}+\sigma_{s}^{2}}} \\
& =\frac{\frac{660-25464.79619}{\mu_{r}^{2}}}{\sqrt{\frac{(25)^{2}+(988.29986)^{2}}{\mu_{r}^{4}}}}=3.09 \\
& \mu_{r}^{4}-78.23788 \mu_{r}^{2}+1487.62018=0 .
\end{aligned}
$$


TABLE III

REQUIRED RADII FOR DIFFERENT METHODS (MM)

\begin{tabular}{c|c|c|c|c|c|r}
\hline & \multicolumn{7}{|c}{$\sigma_{h}(\mathrm{MPa})$} \\
\hline & 0 & 2.5 & 5 & 10 & 12.5 & 15 \\
\hline Safety Factor design method & \multicolumn{7}{|c|}{10.8} \\
$\begin{array}{c}\text { Reliability design method (Load- } \\
\text { strength interference) }\end{array}$ & \multicolumn{7}{|c|}{6.8} \\
\hline New method in this paper & 6.8 & 7.5 & 8.0 & 10.9 & 14.5 & 33.1 \\
\hline
\end{tabular}

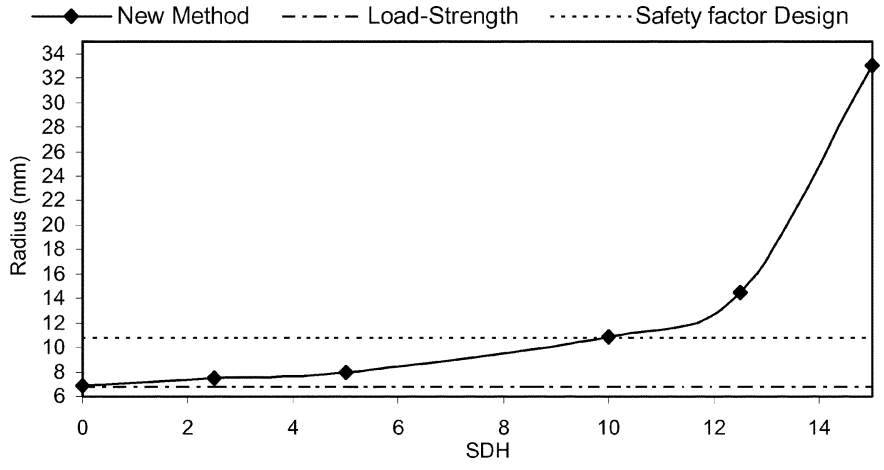

Fig. 15. Radius compared with different methods.

The mean value of the bar can then be obtained by solving (41), which gives

$$
\mu_{r}=6.75657(\mathrm{~mm}) \approx 6.8(\mathrm{~mm}) .
$$

2) Design Using the Material Failure Theory With Applied Safety Factor: To compare the results, the bar material and load remain as in Table I. Here the safety factor is set to $n=3$, and then we have

$$
\sigma=\frac{\mu_{l}}{\pi \cdot r^{2}}=\frac{80000}{\pi \cdot r^{2}} \leq[\sigma]=\frac{\mu_{\delta}}{n}=\frac{660}{3}=220(\mathrm{MPa}) .
$$

From the above equation, the bar radius is computed as

$$
r \geq 10.75867(\mathrm{~mm}) \approx 10.8(\mathrm{~mm}) \text {. }
$$

3) Design Using the New Method Presented in This Paper: To investigate the effect of SDH on the bar design, SDH will be set as $0,2.5,5,10,12.5$, and 15. Fig. 14 illustrates the relationship between the reliability and the bar radius with different $\sigma_{h}$.

Using Fig. 14, the required bar radius for the required reliability of 0.999 is obtained for each $\sigma_{h}$, the values of which are given in Table III. Fig. 15 plots the values of the required radii as a function of SDH. When $\sigma_{h}$ increases from 0 to 15 , the required radius increases greatly from 6.8 to 33.1 .

4) Comparison of Results: To achieve a reliability of 0.999 , the following designs are listed in Table III:

1) conventional load-strength interference theory, radius $=$ $6.8 \mathrm{~mm}$

2) safety factor method, radius $=10.8 \mathrm{~mm}$ for a safety factor of 3 .

3) Monte-Carlo method,

Radius $=6.8 \mathrm{~mm}$ for the homogeneous case, $\sigma_{h}=0$

Radius $=14.5 \mathrm{~mm}$ for $\sigma_{h}=12.5$, and

Radius $=33.1 \mathrm{~mm}$ for $\sigma_{h}=15$.

Comparing the calculated values of the required bar radius, when $\sigma_{h}$ is equal to zero (the material is homogenous), the size of the bar radius from the new method is the same as the one from the conventional load-strength interference method.

The radius calculated using the safety factor method depends on the value of the safety factor chosen. In this example, a safety factor of 3 was used. It can be seen that, if the material is inhomogeneous with $\sigma_{h}>10$, then the safety factor method with $\mathrm{SF}=3$ underestimates the required radius of the bar.

Therefore, the method proposed in this paper gives results which are comparable with conventional load-strength interference, and the safety factor methods. However, it does have the advantage of generating specific results which relate to the inhomogeneity present via the use of a standard deviation of material strength related to material inhomogeneity.

\section{SUMmARY AND CONCLUSIONS}

A method of analysis is proposed which takes into account material inhomogeneity such that the reliability of components of different sizes, but subject to the same applied stress, can be calculated. The method is a development of conventional load-strength interference theory. The proposed method is based on a Monte-Carlo method; and the results have been compared to both the conventional load-interference theory, and a calculation method based on nominal stress, plus a safety factor. If the material is assumed to be homogeneous, then the new method gives the same results as the conventional load-interference method. If the material is assumed not to be homogeneous, then the new method calculates that larger dimensioned components are required to achieve a desired reliability. Unless large safety factors are chosen, then the safety factor method will underestimate the size of components for high reliability.

The method provides an opportunity to explore the effect of component volume on reliability calculations. For key components, the method provides an opportunity for designers and reliability engineers to undertake sensitivity analyses concerning the possible effect of component size on reliability for different degrees of material inhomogeneity. To calculate specific values of reliability, the method requires material data which is not recorded generally at the present time. However, it is quite feasible to obtain the data by a test program, say in the case of certain safety critical components.

\section{REFERENCES}

[1] I. Bazovski, Reliability Theory and Practice. Englewood Cliff, New Jersey: Prentice-Hall, 1961.

[2] T. R. Moss, Mechanical Reliability -Research Needs: 12th ARTS, University of Manchester, 1996

[3] M. Rausand and K. Øien, "The basic concepts of failure analysis," Reliab. Eng. Syst. Saf., no. 53, pp. 73-83, July 1996.

[4] J. A. Collins, Failure of Materials in Mechanical Design: Analysis, Prediction, Prevention, Second ed: John Wiley \&Sons, 1993. 
[5] J. Carvill, Mechanical Engineers Data Handbook. Oxford: Butterworth-Heinemann, 1993.

[6] A. D. S. Carter, Mechanical Reliability, Second ed: Macmillan Education LTD, 1986.

[7] J. D. Andrews and T. R. Moss, Reliability and Risk Assessment. London: Longman, 1993.

[8] A. M. Freudenthal, M. Garrelts, and M. Shinozuka, The Analysis of Structure Safety Journal of the Structural Division ASCE, vol. 92, no. ST1, pp. 267-325, 1966.

[9] W. G. Ireson et al., Handbook of Reliability Engineering and Management. New York, NY: McGraw-Hill, 1995.

[10] J. Davidson, The Reliability of Mechanical Systems. London: Paston Press, 1988.

[11] W.-X Liu, Mechanical Reliability Design. Beijing: Hsinghua University Press, 1996.

[12] Q.-L Zhou et al., Reliability and Maintenance Engineering: Hebei Education Press, 1992.

[13] P. Martin, "A review of mechanical reliability," Proc. I MECH E Part E Journal of Process Mechanical Engineering, vol. 212, pp. 281-287, November 1998.

[14] D. Kececioglu and D. Cormier, "Designing a specified reliability directly into component," in Proc. 3rd Annu. Aerospace Reliability and Maintainability Conf., 1964, pp. 564-565.

[15] G. Thompson, Improving Maintainability and Reliability Through Design. London, UK: Professional Engineering Publications, 1999.

[16] J.-P Li and G. Thompson, "Investigating effects of volume of mechanical components on structure reliability," in European Safety and Reliability Conf., Maastricht, The Netherlands, June 2003, pp. 1025-1029.
[17] H.-Z. Wang and H. Pham, "Survey of reliability and availability evaluation of complex networks using Monte Carlo techniques," Microelectron. Reliab., vol. 37, no. 2, pp. 187-209, Feb. 1997.

[18] J. M. Gere and S. P. Timoshenko, Mechanics of Materials, Fourth ed: International Thomson Publishing, 1997.

Jian-Ping Li is an Associate Professor in Shijiazhuang Institute of Science and Technology, P.R. China. He is a senior member of the China Mechanical Engineering Society. Now, He is working as a Research Assistant and studying for his $\mathrm{Ph} . \mathrm{D}$. degree in Mechanical Failure Modeling in a virtual reality environment at UMIST, U.K. From 2000-2001, he was visiting at Cambridge University. He received his B.Sc. in Mechanical Engineering in 1986 from Shijiazhuang Institute of Science and Technology, and his M.Sc. in Mechanical Optimization Design in 1989 from Nanjing Institute of Science and Technology. He has published more than 60 papers. His main research interests lie in modeling of stochastic systems, system reliability and maintainability, and the theory and application of advanced optimization methods (including genetic algorithms).

G. Thompson is a Professor and Head of the Mechanical, Aerospace and Civil Engineering School, Manchester University, U.K. and Adjunct Professor at Department of Mechanical, Aerospace and Manufacturing Engineering, Luleå Technical University, Luleå, Sweden. 